\title{
Correction to: Participatory Data Physicalization: A New Space to Inform
}

Matteo Moretti and Alvise Mattozzi

Correction to:

Chapter "Participatory Data Physicalization:

A New Space to Inform" in: E. Cicalò (Ed.):

Proceedings of the 2nd International and Interdisciplinary

Conference on Image and Imagination, AISC 1140, https://doi.org/10.1007/978-3-030-41018-6_86

In the original version of the book, the following belated correction has been incorporated: The abstract has been revised in Chapter 86 . 